Recepción: 15 / 04 / 2017

Ciencias Administrativas

Aceptación: 01 / 05 / 2017

Articulo Científico

Publicación: 15 / 05 / 2017

\title{
Implementación de un modelo para elaborar planes de negocios para las PYMES acorde a las exigencias de los organismos de crédito
}
Implementation of a model to develop business plans for PYMES according to the requirements of credit agencies

\section{Implementação de um modelo para o desenvolvimento de planos de negócios para as PYMES de acordo com as exigências de agências de crédito}

Ronny F. Onofre-Zapata ronofre@utb.edu.ec

Carlos G. Aguirre-Rodriguez caduirre@utb.edu.ec

Carlos L. Navas-Chancay "' carlosluis.navas@hotmail.es

Correspondencia: ronofre@utb.edu.ec

Magister en Administración de Empresas; Diploma Superior en Docencia Universitaria; Ingeniero Comercial, Universidad Técnica de Babahoyo, Ecuador.

II. Magister en Docencia y Currículo; Magister Ejecutivo en Dirección de Empresas con Énfasis en Gerencia Estratégica; Especialista en Gerencia de Proyectos; Diplomado Superior en Gerencia de Marketing; Ingeniero Industrial; Universidad Técnica de Babahoyo, Ecuador.

III. Magister en Gerencia de Servicios de Salud; Magister en Docencia y Currículo; Diploma Superior en Gestión de Desarrollo de los Servicios de Salud; Especialista en Oftalmología; Doctor en Medicina y Cirugía; Universidad Técnica de Babahoyo, Ecuador . 


\section{Resumen}

A pesar de enfrentar problemas importantes inherentes a la propia peculiaridad organizacional y las limitaciones en el otorgamiento de crédito para las pequeñas y medianas empresas, hoy por hoy es un mercado en proceso de recuperación, iniciado por el actual gobierno en a través de programas de incentivos, como por ejemplo el crédito 5 - 5 (cinco años de plazo a 5\% de interés).

Bajo este contexto el objetivo que se persigue con nuestra propuesta es la modelización de un plan de negocio que le permita a la Pyme, en primer lugar desarrollarse organizacionalmente, optimizando sus recursos y por ende la mejora en su rentabilidad, en segundo lugar asegurar su permanencia en el mercado.

Basados en la información obtenida en las investigaciones y maximizar la compresión de nuestra propuesta, se diseñaron tres escenarios, que consisten en:

Conceptualización de los componentes de un plan de negocio.

Aplicación de los componentes en una Pyme que actualmente compite en el mercado de la informática.

Compartir los conocimientos y lecciones aprendidas, con las Pymes afiliadas en las Cámaras de Comercio y Pequeña Industria localizadas en la ciudad de Babahoyo, a través de cursos y seminarios que se impartirían durante el primer trimestre del año 2011.

Palabras clave: PYMES; organismos de crédito; rentabilidad. 


\begin{abstract}
Despite facing important problems inherent to the own organizational peculiarity and the limitations in the granting of credit for the small and medium companies, today it is a market in process of recovery, initiated by the present government in through programs of incentives, Such as credit 5 5 (five-year term at $5 \%$ interest).
\end{abstract}

In this context, the objective pursued with our proposal is the modeling of a business plan that allows the SME, in the first place to develop organizationally, optimizing its resources and therefore the improvement in its profitability, secondly to ensure its permanence in the market.

Based on the information obtained in the research and maximize the compression of our proposal, three scenarios were designed, which consist of:

Conceptualization of the components of a business plan.

Application of the components in a PYMES that currently competes in the computer market.

Share knowledge and lessons learned with affiliated SMEs in the Chambers of Commerce and Small Industry located in the city of Babahoyo, through courses and seminars that would be given during the first quarter of 2011.

Key words: PYMES; Credit institutions; cost effectiveness. 


\section{Resumo}

Apesar de enfrentar peculiaridade organizacional próprios problemas e limitações significativas inerentes nos empréstimos às pequenas e médias empresas, hoje é um processo de recuperação do mercado, iniciada pelo atual governo através de programas de incentivo tais como crédito (prazo de cinco anos a juros de 5\%) 5-5.

Neste contexto, o objectivo prosseguido pela nossa proposta é a modelagem de um plano de negócios que permitirá que as PME, desenvolvido pela primeira vez organizacional, otimizando os recursos e melhorar a rentabilidade, em segundo lugar garantir a sua permanência no mercado.

Com base em informações obtidas em investigações e maximizar a compressão da nossa proposta, três cenários foram desenhados, composta por:

Conceptualização dos componentes de um plano de negócios.

Aplicação dos componentes em uma PYMES actualmente a competir no mercado de computação.

Partilha de conhecimentos e lições aprendidas, com filiais PME no Câmaras de Comércio e Indústria Pequenas localizados na cidade de Babahoyo, por meio de cursos e seminários que seria realizado durante o primeiro trimestre de 2011.

Palavras chave: PYMES; agências de crédito; rentabilidade. 


\section{Introducción.}

Las contradicciones propias de las sociedades no industrializadas, en las que el crecimiento de la población económicamente activa supera con creces los medios de producción disponibles, han llevado a generar nuevas formas productivas, conocidas como Pymes las que se han distinguido por una gran diversidad de iniciativas realizadas por una pluralidad de actores.

El desarrollo de esta investigación tiene por objeto llegar a conocer las características de las

Pymes en la ciudad de Babahoyo, de las cuales lamentablemente no existe información; para con ello poder establecer un diagnóstico situacional, partiendo de condiciones muy evidentes que identifican la debilidad de este sector y que afectan seriamente el desarrollo y permanencia de estas empresas en el mercado local.

Además, a través del análisis de los resultados, proponer y desarrollar una solución viable que contribuya, llevando líneas de acción fundamentales ante las necesidades de este importante sector económico.

Esperamos contribuir con una guía de consulta que revele la realidad de las Pymes en la ciudad de Babahoyo, y que demuestre que indudablemente hay mucho por hacer en este campo que constituye una valiosa parte de la economía, y necesita el apoyo del Estado para potenciar su capacidad de generar empleo, reducir la pobreza y mejorar la distribución del ingreso.

El presente estudio busca realizar un análisis de las Pymes para diseñar un modelo de plan de negocio, que permita cubrir las exigencias de los organismos de crédito y las necesidades del desarrollo empresarial. 


\section{Materiales y métodos}

Es evidente que la crisis financiera internacional, de la mano con cierta incertidumbre local que nace de la administración del actual gobierno, ha producido una serie de sucesos que dejan a la luz pública cambios sustanciales en el acontecer económico nacional. Uno de estos cambios es el emprendimiento nacido de la necesidad de producir dinero y de la escasez de contratación de personal en la empresa privada.

De acuerdo a información obtenida de la Superintendencia de Compañías, y la Federación Nacional de Cámaras de la Pequeña Industria (FENAPI), existen un poco más 600.000 pequeñas y mediana empresas (Pymes) en el Ecuador y se cree muy probable que debido a los actuales niveles de desempleo esta cifra ascienda en el presente año. Estas empresas emplean alrededor de 1'800.000 personas, es decir, alrededor del 38\% de la ocupación total del país.

El número actual de Pymes establecidas en el país representan una cantidad considerable, es importante recalcar que al final del año no todas ellas percibirán los resultados financieros esperados, desapareciendo unas, otras con pequeñas posibilidades de continuar en el mercado y otras manteniendo la liquidez suficiente para seguir desarrollando sus actividades normales.

La forma tradicional o precaria que se sigue para establecer una Pyme, es un aspecto muy negativo, que probablemente causará problemas que pueden llevar hasta el cierre de la empresa. Es muy común observar cómo se acusa a acontecimientos externos de ser los causantes de diferentes problemas que ocurren al interior de una empresa, dejando a un lado, o ni siquiera considerando, que los problemas pueden ser fácilmente producto de una mala gestión administrativa interna. 
Las principales causas de que gran parte de las Pymes presenten inconvenientes al corto o mediano plazo, es que no existe un estudio antes de su implementación y además no trabajan bajo ninguna estructura organizada que les permita ejecutar sus actividades cotidianas, siguiendo procesos previamente estudiados y analizados, cuyo orden permita llegar a las metas establecidas de una manera eficaz y eficiente, con un mínimo de error y controlando sus procesos en puntos estratégicos.

En el entorno empresarial existen una serie de sugerencias acerca de herramientas de organización administrativa, que han sido previamente estudiadas, aplicadas y cuyos resultados han sido muy positivos para la empresas. Estas herramientas estandarizadas hacen a un lado la administración tradicional, y le abren paso a la gestión de calidad

A continuación reproducimos las características de las pymes en nuestro país, basados en información obtenida en el Ministerio de Comercio Exterior, Industrialización Pesca y Competitividad:

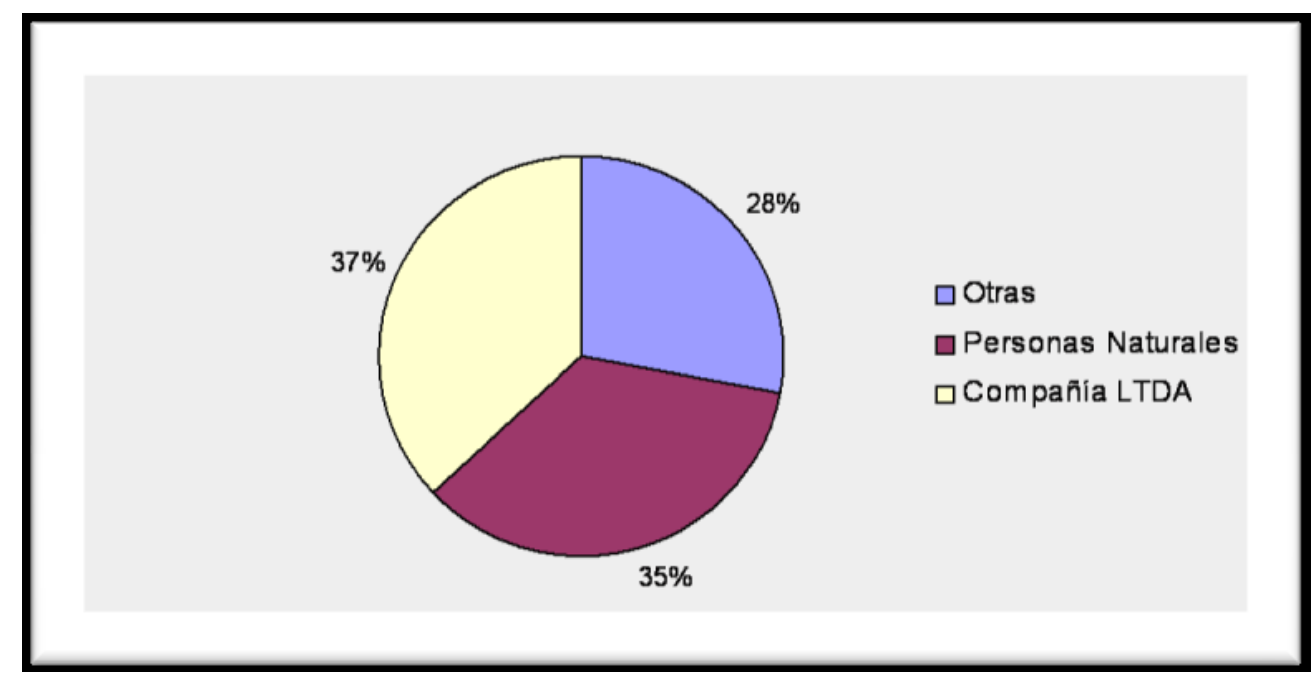

Organización Jurídica de las PYMES

Fuente: Ministerio de Comercio Exterior, Industrialización Pesca y Competitividad 
La información nos indica que en la organización jurídica de la pequeña y mediana empresa prevalecen las compañías limitadas $(37.3 \%)$ y que tienen un peso muy significativo aquellas que operan como personas naturales $(35.2 \%)$, de lo cual se concluye que en la conformación del capital de la pequeña industria, se mantiene todavía una estructura cerrada o de tipo familiar.

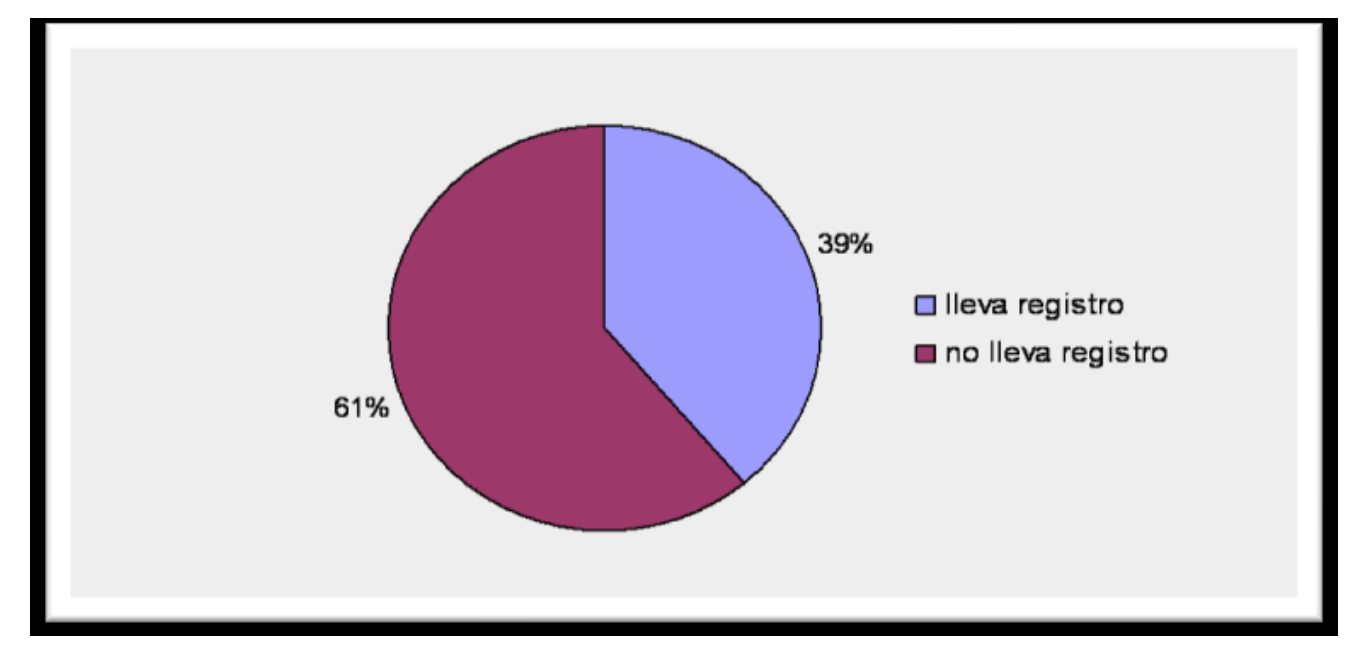

\section{Contabilidad de las PYMES}

\section{Fuente: Ministerio de Comercio Exterior, Industrialización Pesca y Competitividad}

En relación con los registros contables por parte de las Pymes, apenas el 39\% de las mismas llevan registros, mientras que el $61 \%$ restante no lo hace. Además de este hecho, cabe indicar que del $39 \%$ que los lleva, el $87 \%$ lo hace por cuenta propia y el $13 \%$ lo hace por cuanto es un requerimiento legal para la realización de sus actividades normales. Este hecho indica que no existe una política contable suficientemente específica para este tipo de empresas. 


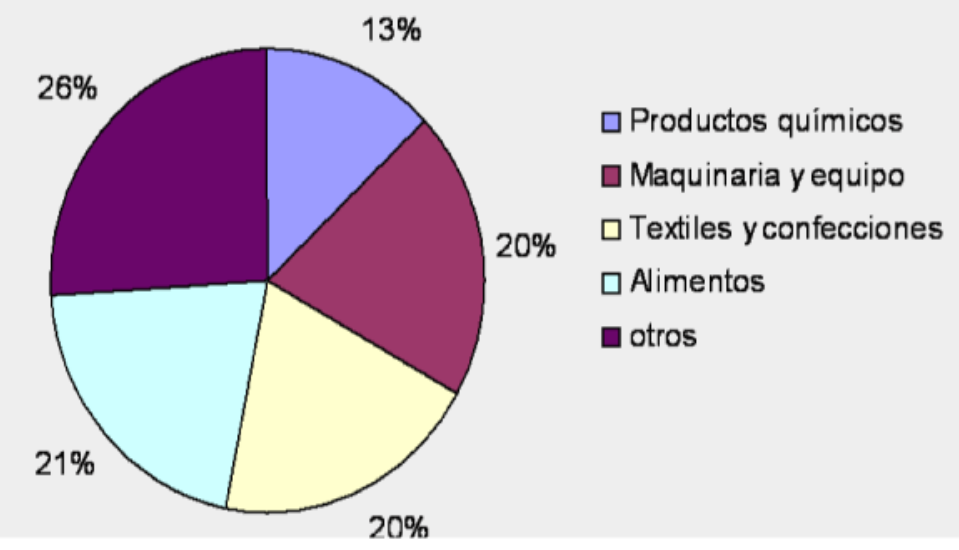

Empleo generado por las PYMES

Fuente: Ministerio de Comercio Exterior, Industrialización Pesca y Competitividad

Con respecto al empleo, los sectores de alimentos (20.7\%), textil y confecciones (20.3\%), maquinaria y equipo (19.9\%) y productos químicos (13.3\%), generan el $74 \%$ de plazas de trabajo de las Pymes. A través de la información obtenía, se ha podido determinar que el promedio de empleo es de 19 personas por empresa y que las mujeres representan el 33\% del total de ocupados.

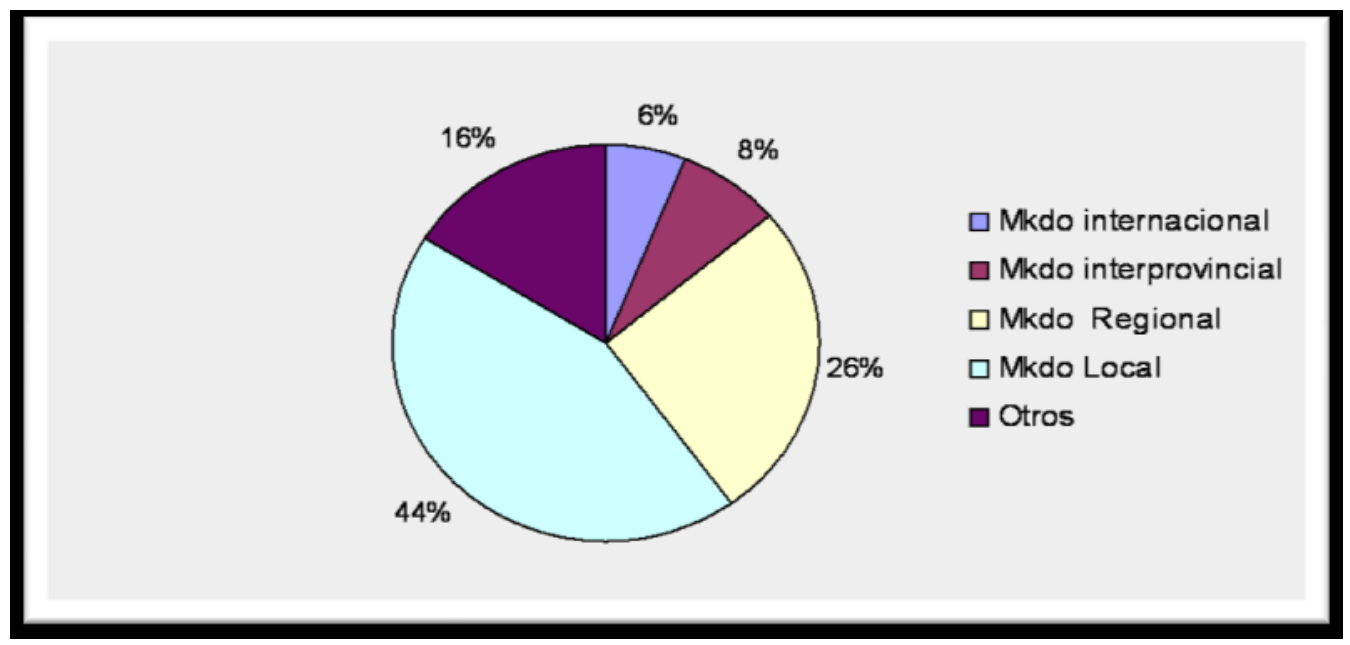

Mercado de las PYMES

Fuente: Ministerio de Comercio Exterior, Industrialización Pesca y Competitividad 
En cuanto al mercado, para el $44 \%$ de las empresas el mercado es básicamente local (su ciudad) mientras que para el $26 \%$ sería de alcance regional (ciudad, provincia de origen y provincias circunvecinas). El $8 \%$ habría extendido su radio de acción a otras provincias (interprovincial) y solamente el $6 \%$ estaría sosteniendo el flujo exportable.

Las Pymes no exportan actualmente de manera creciente y sostenida, debido a que tienen ciertas dificultades para hacerlo: capacidad insuficiente para atender grandes volúmenes, desconocimiento de los requisitos nacionales e internacionales, precios no competitivos, limitaciones en calidad y trabas arancelarias y no arancelarias que imponen otros países.

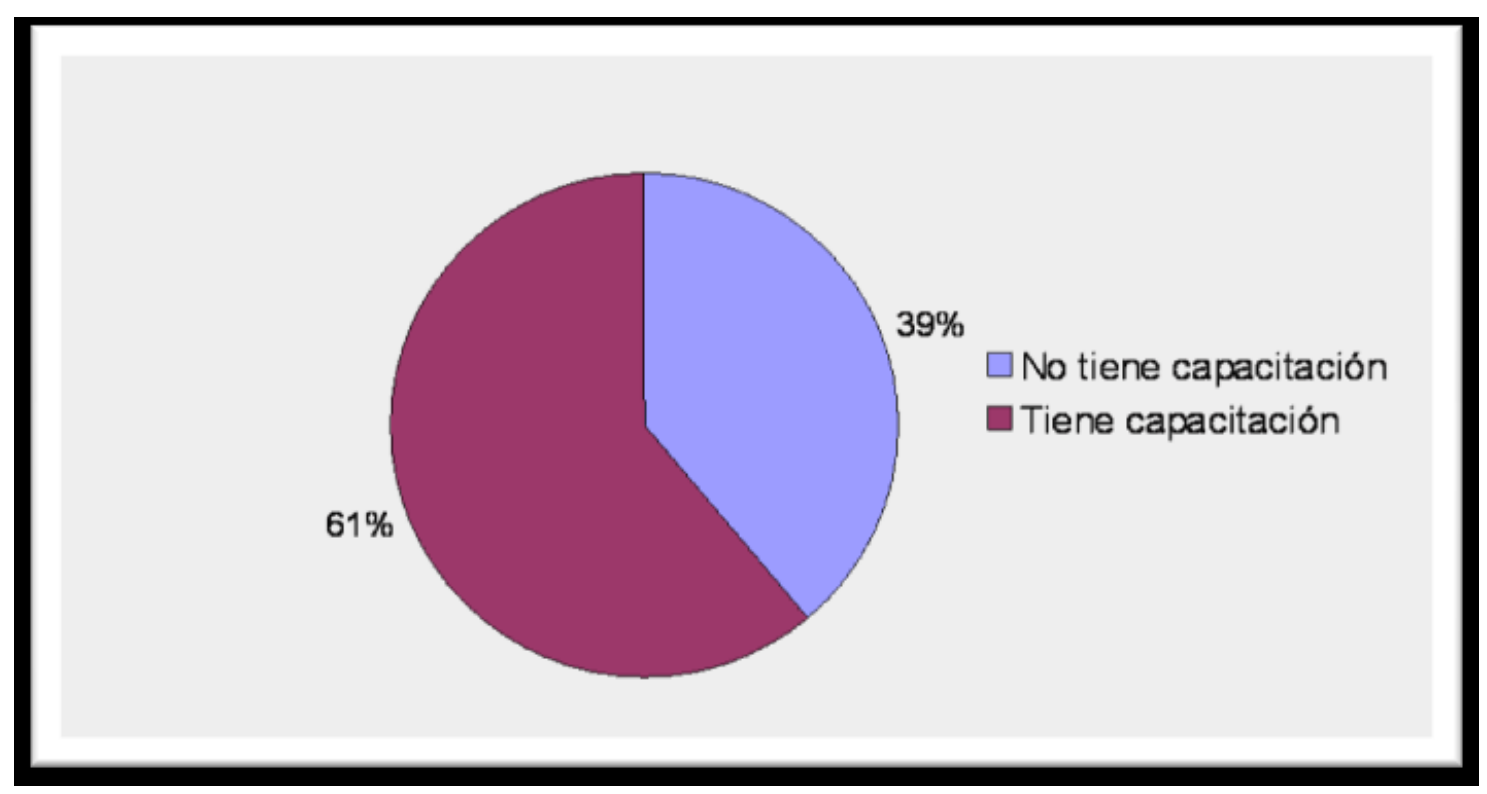

Capacitación de las PYMES

\section{Fuente: Ministerio de Comercio Exterior, Industrialización Pesca y Competitividad}

La capacitación dentro de las Pymes representa un valor considerable, aunque no satisfactorio el porcentaje del 60.8\%, lo cual implica que existe un 39.2\% de las mismas que aún no consideran la importancia de la capacitación de su personal.

\section{Situación problemática}


Con el inicio de la explotación petrolera en 1972, el endeudamiento externo se profundizó, el estado creció convirtiéndose en la principal fuente de empleo de la sociedad ecuatoriana, además las industrias amparadas por las leyes de fomento, subsidios estatales, estímulos tributarios y programas de crédito dirigido, también crearon plazas de trabajo. Paralelamente a este escenario promovido por la política vigente que aquel entonces, los talleres artesanales, pequeños negocios, entre otros, continuaron laborando con su tradicional esquema, anexando en forma ágil y oportuna algunas modificaciones como producto de las mejoras en los servicios de energía eléctrica, ampliación de la red vial y de la telefonía, la presencia de la televisión y los electrodomésticos, permitieron un significativo grado de modernización a las pymes, para facilitar su trabajo.

El uso de "la máquina de coser eléctrica fue incorporada por sastres y modistas a sus talleres, lo que les permitió mejorar la calidad y rendimiento, con el consiguiente crecimiento de producción y productividad, sobre todo cuando fue posible adquirir maquinaria complementaria como: overlock, ojaladora, pretinadora, cortadora eléctrica, etc.

Cosa similar sucedió con los restaurantes y puestos de comida rápida, cuando el gas de uso doméstico sustituyó a los fogones de leña y carbón, el reverbero a gasolina o la cocineta a kerex. Del mismo modo, las salas de belleza con las secadoras eléctricas; los talleres de cerrajería con las nuevas soldadoras y esmeriles; las carpinterías con las sierras, canteadoras, perforadoras,cepilladoras, tornos, lijadoras; las tiendas de barrio con refrigeradora y teléfonos a disposición de los clientes; los bazares con las fotocopiadoras.”(Alarcón C, 2006)

Sería muy larga la enumeración detallada del impacto de las nuevas tecnologías en cada taller productivo o establecimiento de servicio. Cabe destacar que la modernización no desplazó ni 
eliminó a la pyme, simplemente readecuó su participación en el mercado con algunas necesarias innovaciones, pues la pequeña y mediana empresa de larga trayectoria histórica en nuestra sociedad cambio las herramientas pero no la naturaleza emprendedora del ser humano.

Cuando el gobierno ecuatoriano implanto el modelo económico neoliberal este empezó a reducir el tamaño del Estado, terminar los subsidios, propiciar la privatización de las áreas en las que se había realizado inversión pública, promover la apertura a la inversión extranjera, impulsar el libre comercio internacional y la imposición periódica de medidas de ajuste, más conocidas con el nombre de paquetazos económicos. Esas políticas generaron más pobreza, desempleo y una grave profundización de la brecha entre los sectores sociales.

Luego de que el estado fue el principal empleador, se emprendió un agresivo plan de reducción de su tamaño, a través de la eliminación del número de empleados públicos, utilizando como mecanismos: la supresión de partidas presupuestarias, la no renovación de contratos y la compra de renuncias. Mediante estos procedimientos, se disminuyó, al menos 10.000 empleos públicos en la década de los 90.

La modernización y privatización en el sector público, así como la incorporación de modernas tecnologías en la empresa privada, causaron que muchas personas pierdan su empleo o no encuentren con posibilidades de incorporarse activamente al mercado laboral, creando nuevas unidades económicas de subsistencia llamadas Pymes.

A las Pymes se las define como un conjunto de pequeñas y medianas empresas que se miden de acuerdo al volumen de ventas, el capital social, el número de personas ocupadas, el valor de la producción o el de los activos. Así también se toma como referencia el criterio económico y el nivel tecnológico. 
Implementación de un modelo para elaborar planes de negocios para las PYMES acorde a las exigencias de los organismos de crédito

Las Pymes pueden dedicarse a actividades diversas, entre las cuales se pueden mencionar:

Agricultura, caza, pesca.

Exploración de minas y cantera.

Industrias manufactureras.

Construcción.

Comercio al por mayor y al por menor, restaurantes y hoteles.

Transporte, almacenamiento, y comunicaciones.

Establecimientos financieros, seguros, bienes inmuebles y servicios prestados a las empresas.

Servicios comunales, sociales y personales.

Se las considera como una organización económica donde se combinan los factores productivos (naturaleza, trabajo y capital) para generar los bienes y servicios que una sociedad necesita para poder satisfacer sus necesidades, por lo que se convierte en el eje de la producción.

\section{El desarrollo de las Pymes en el Ecuador}

La creación de las Pymes se desarrolla en base a una idea, que se da como consecuencia de la detección de una oportunidad de negocio. Son muchos los factores que pueden llevar a una persona a inclinarse por un negocio concreto, los factores son:

Repetición de experiencias ajenas.

Nuevas oportunidades de negocio en mercados poco abastecidos, de nueva creación o con un alto porcentaje de crecimiento.

Conocimientos técnicos sobre mercados, sectores o negocios concretos. 
La experiencia del futuro empresario, que ha sido trabajador o directivo de otro negocio y que pretende independizarse.

La importancia de las Pymes en la economía del Ecuador se basa en:

Asegura el mercado de trabajo mediante la descentralización de la mano de obra, lo cual se lo considera necesario para el correcto funcionamiento del mercado laboral.

Tienen efectos socioeconómicos importantes ya que permiten la concentración de la renta y la capacidad productiva desde un número reducido de empresas hacia uno mayor.

Reducen las relaciones sociales a términos personales más estrechos entre el empleador y el empleado, en general, sus orígenes son unidades familiares.

Presentan mayor adaptabilidad tecnológica y menor costo de infraestructura.

En la ciudad de Babahoyo se identificó que existe en las Pymes un alto nivel de informalidad en el marco legal, generalmente no cuentan con las licencias, no registran a sus empleados en la seguridad social, ni reportan sus ventas a la autoridad impositiva, esto se debe en parte a que los costos asociados a registrarse son altos y también porque el proceso es complicado y tedioso.

Indagando sobre las fuentes de financiamiento para iniciar el negocio se descubre un panorama interesante. La mayoría de las Pymes inician su empresa gracias a sus ahorros personales. La segunda fuente de financiamiento son los préstamos de familiares y amigos. Muy pocos de los entrevistados indicaron no conocer alguna institución financiera. Sin embargo, es muy bajo el nivel de financiamiento de las Pymes a través del sector formal de instituciones financieras. 
Las Pymes en esta ciudad funcionan bajo estándares administrativos tradicionales, donde la informalidad es la manera natural de desarrollar las actividades cotidianas. En general no existe un sistema que organice cada una de las áreas y actividades que se realizan, los procedimientos de trabajo no están establecidos, los controles son inexistentes o poco efectivos en su función, la eficiencia y eficacia del trabajo no son medibles, no hay seguimiento de actividades que permita encontrar mejoras constantes a las mismas y, entre otras cosas, se reduce la administración al simple manejo de los ingresos y egresos, y a la delegación de actividades de acuerdo al criterio de la persona al mando.

\section{Resultados.}

A continuación, los resultados de la investigación realizada en la ciudad de Babahoyo; a través de encuestas, dirigidas a los propietarios de las Pymes.

\section{PREGUNTA 1}

Número de empleados que laboran en la Pyme 
Tabla de frecuencias

\begin{tabular}{|c|c|c|c|}
\hline $\begin{array}{r}\text { Numero } \\
\text { empleados }\end{array}$ & $\begin{array}{l}\text { Frecuencia } \\
\text { absoluta }\end{array}$ & $\begin{array}{l}\text { Frecuencia } \\
\text { relativa } \\
\end{array}$ & $\begin{array}{l}\text { Frecuencia } \\
\text { relativa } \\
\text { acumulada }\end{array}$ \\
\hline 1 a 2 & 61 & $42,07 \%$ & $42,07 \%$ \\
\hline 3 a 4 & 47 & $32,41 \%$ & $74,48 \%$ \\
\hline 5 a 6 & 12 & $8,28 \%$ & $82,76 \%$ \\
\hline 7 a 8 & 7 & $4,83 \%$ & $87,59 \%$ \\
\hline 9 a 10 & 6 & $4,14 \%$ & $91.73 \%$ \\
\hline 11 a 12 & 4 & $2,76 \%$ & $94.49 \%$ \\
\hline 13 a 14 & 3 & $2,07 \%$ & $96.56 \%$ \\
\hline 15 a 16 & 4 & $2,76 \%$ & $99.32 \%$ \\
\hline Más de 15 & 1 & $0,69 \%$ & $10.00 \%$ \\
\hline TOTAL & 145 & $100,00 \%$ & \\
\hline
\end{tabular}

El 74,48\% de las Pymes de la ciudad de Babahoyo tienen un rango de entre uno a cuatro empleados, el 4.14\% de las Pymes visitadas cuentan con hasta diez empleados, y apenas el 0,69\% del total de la muestra tienen más de 15 empleados.

\section{PREGUNTA 2}

¿Usted alguna vez ha escuchado sobre los préstamos o créditos que otorga el sector financiero? 
Tabla de frecuencias

\begin{tabular}{|c|c|c|}
\hline & $\begin{array}{c}\text { Frecuencia } \\
\text { absoluta }\end{array}$ & $\begin{array}{c}\text { Frecuencia } \\
\text { relativa }\end{array}$ \\
\hline $\mathrm{Si}$ & 89 & 61.38 \\
\hline No & 56 & 38,62 \\
\hline TOTAL & 145 & $100,00 \%$ \\
\hline
\end{tabular}

Un importante $61,38 \%$ de los entrevistados si conoce que los organismos financieros otorgan créditos, pero es alarmante que el 38,62\% de los microempresarios no conozcan de los créditos que otorgan el sistema financiero.

\section{PREGUNTA 3}

¿Sabía usted que para agilizar los trámites para los préstamos, los organismos financieros solicitan planes estratégicos de negocios?

Tabla de frecuencias

\begin{tabular}{|c|c|c|}
\hline & $\begin{array}{c}\text { Frecuencia } \\
\text { absoluta }\end{array}$ & $\begin{array}{c}\text { Frecuencia } \\
\text { relativa }\end{array}$ \\
\hline $\mathrm{Si}$ & 19 & $13,10 \%$ \\
\hline No & 126 & $86,90 \%$ \\
\hline TOTAL & 145 & $100,00 \%$ \\
\hline
\end{tabular}

El $86,90 \%$ de los entrevistados no conocen que hay que elaborar planes de negocios para solicitar crédito en las instituciones financieras y un $13,10 \%$ si conocen. 


\section{PREGUNTA 4}

¿Conoce usted de alguna asociación que otorgue créditos a sus asociados?

Tabla de frecuencias

\begin{tabular}{|c|c|c|}
\hline & $\begin{array}{c}\text { Frecuencia } \\
\text { absoluta }\end{array}$ & $\begin{array}{l}\text { Frecuencia } \\
\text { relativa }\end{array}$ \\
\hline $\mathrm{Si}$ & 2 & $1,38 \%$ \\
\hline No & 143 & $98,62 \%$ \\
\hline TOTAL & 145 & $100,00 \%$ \\
\hline
\end{tabular}

El 98.62\% no conocen de alguna asociación que otorgue crédito y el 1.38\% si conocen.

\section{PREGUNTA 5}

Le gustaría recibir crédito por parte del sector financiero para cubrir sus necesidades que tiene su negocio?

Tabla de frecuencias

\begin{tabular}{|c|c|c|}
\hline & $\begin{array}{c}\text { Frecuencia } \\
\text { absoluta }\end{array}$ & $\begin{array}{c}\text { Frecuencia } \\
\text { relativa }\end{array}$ \\
\hline $\mathrm{Si}$ & 110 & $75,86 \%$ \\
\hline No & 35 & $24,14 \%$ \\
\hline TOTAL & 145 & $100,00 \%$ \\
\hline
\end{tabular}

El $75.86 \%$ de los entrevistados si le gustaría recibir crédito y un $24.14 \%$ no le gustaría. 


\section{PREGUNTA 5}

¿Cómo considera usted que son los trámite de los crédito en el sector financiero?

Tabla de frecuencias

\begin{tabular}{|l|c|c|}
\cline { 2 - 3 } \multicolumn{1}{c|}{} & $\begin{array}{c}\text { Frecuencia } \\
\text { absoluta }\end{array}$ & $\begin{array}{c}\text { Frecuencia } \\
\text { relativa }\end{array}$ \\
\hline Rápido & 35 & $24,14 \%$ \\
\hline Complicado & 110 & $75.86 \%$ \\
\hline \multicolumn{1}{|c|}{ TOTAL } & $\mathbf{1 4 5}$ & $\mathbf{1 0 0 , 0 0 \%}$ \\
\cline { 2 - 3 } & &
\end{tabular}

El $75.86 \%$ de los entrevistados manifestaron que solicitar crédito es complicado y apenas el

$24.14 \%$ consideran que son rápidos.

\section{PREGUNTA 6}

¿Le gustaría recibir información de cómo se solicitan los préstamo o créditos?

Tabla de frecuencias

\begin{tabular}{|c|c|c|}
\hline & $\begin{array}{c}\text { Frecuencia } \\
\text { absoluta }\end{array}$ & $\begin{array}{c}\text { Frecuencia } \\
\text { relativa }\end{array}$ \\
\hline $\mathrm{Si}$ & 129 & $88,97 \%$ \\
\hline No & 5 & $3,45 \%$ \\
\hline $\mathrm{NS} / \mathrm{NC}$ & 11 & $7,59 \%$ \\
\hline TOTAL & 145 & $100,00 \%$ \\
\hline
\end{tabular}


El $88.97 \%$ manifestaron que si les gustaría recibir información y el 3.45\% no están interesados en recibir información, otro $7.59 \%$ son indiferentes.

\section{PREGUNTA 7}

¿Si usted calificara para un crédito del sector financiero en que lo invertiría?

Tabla de frecuencias

\begin{tabular}{|c|c|c|}
\cline { 2 - 3 } \multicolumn{1}{c|}{} & $\begin{array}{c}\text { Frecuencia } \\
\text { absoluta }\end{array}$ & $\begin{array}{c}\text { Frecuencia } \\
\text { relativa }\end{array}$ \\
\hline En compra de artículos que actualmente vendo & 57 & $39,31 \%$ \\
\hline Ampliaría mi negocio con nuevos productos & 45 & $31,03 \%$ \\
\hline Pagaría mis deudas & 43 & $29,66 \%$ \\
\hline TOTAL & $\mathbf{1 4 5}$ & $\mathbf{1 0 0 , 0 0 \%}$ \\
\hline \multicolumn{2}{c|}{} \\
\cline { 2 - 3 }
\end{tabular}

El $70.34 \%$ invertirían el dinero del crédito en desarrollar el negocio y un $29.66 \%$ en pagar sus deudas lo que implica que a corto plazo tendrían problemas para pagar la deuda.

\section{PREGUNTA 8}

¿Alguna vez ha solicitado crédito en alguna institución financiera pública o privada? 


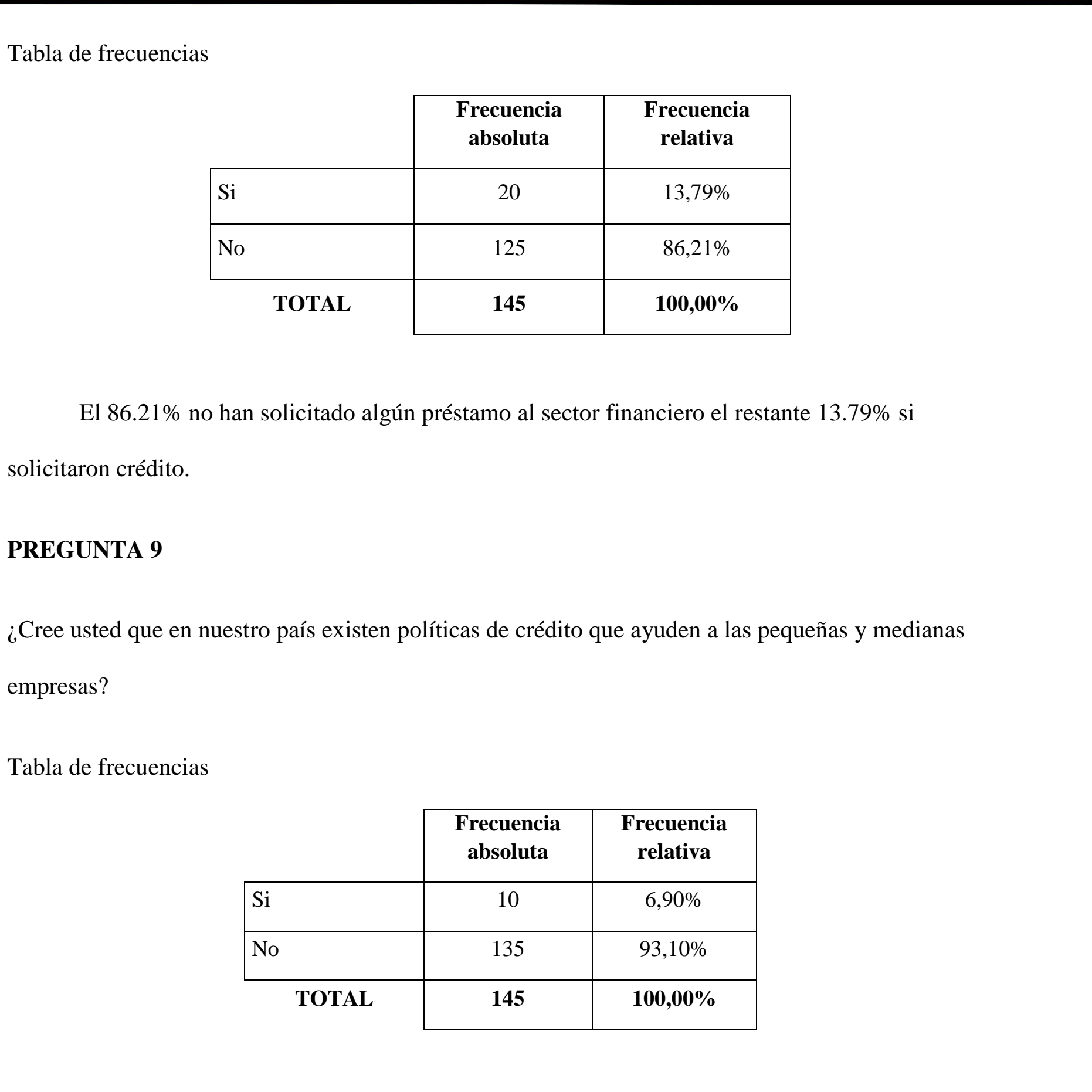

\begin{tabular}{|c|c|c|}
\hline & $\begin{array}{c}\text { Frecuencia } \\
\text { absoluta }\end{array}$ & $\begin{array}{c}\text { Frecuencia } \\
\text { relativa }\end{array}$ \\
\hline $\mathrm{Si}$ & 20 & $13,79 \%$ \\
\hline No & 125 & $86,21 \%$ \\
\hline TOTAL & 145 & $100,00 \%$ \\
\hline
\end{tabular}

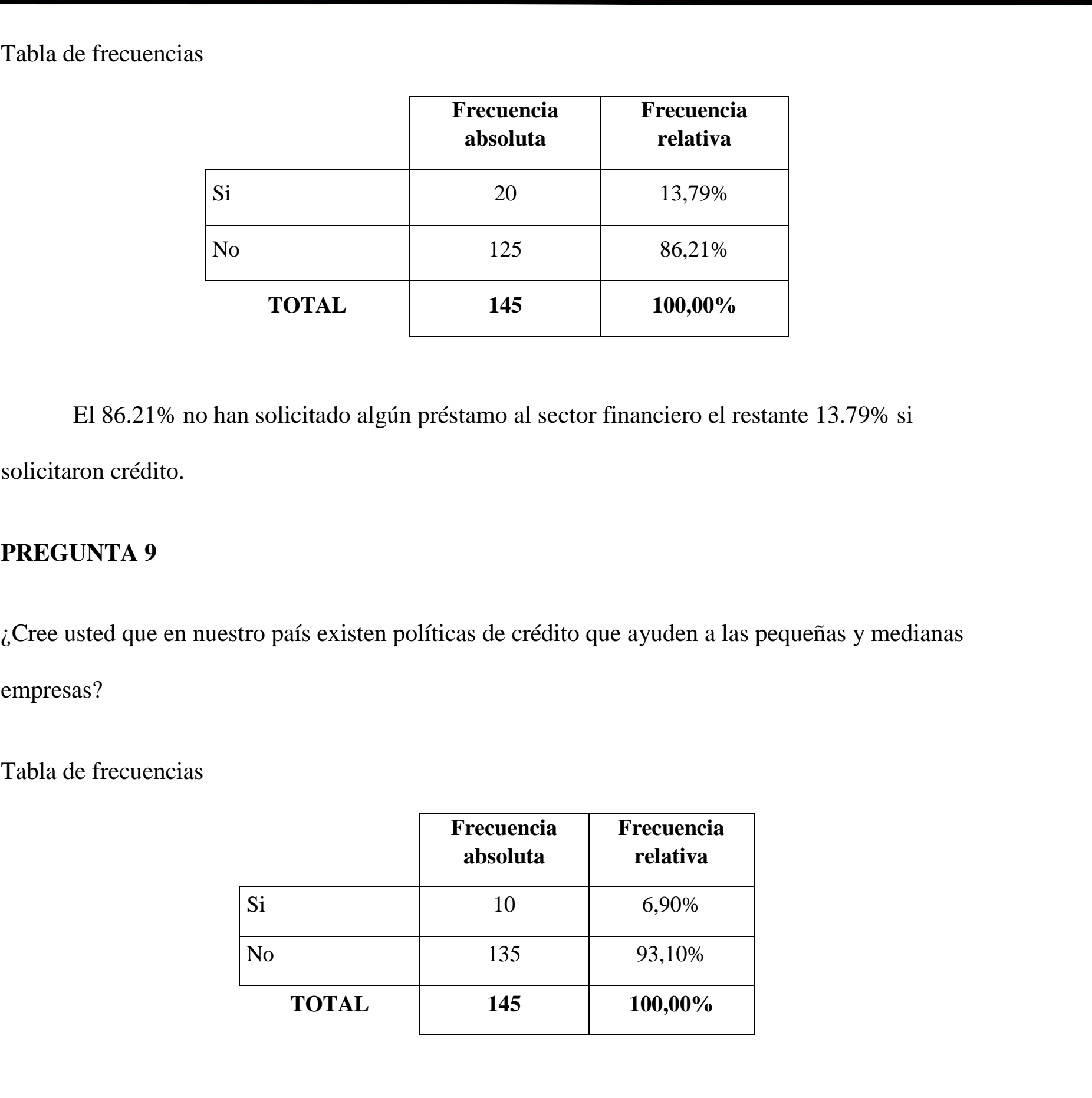

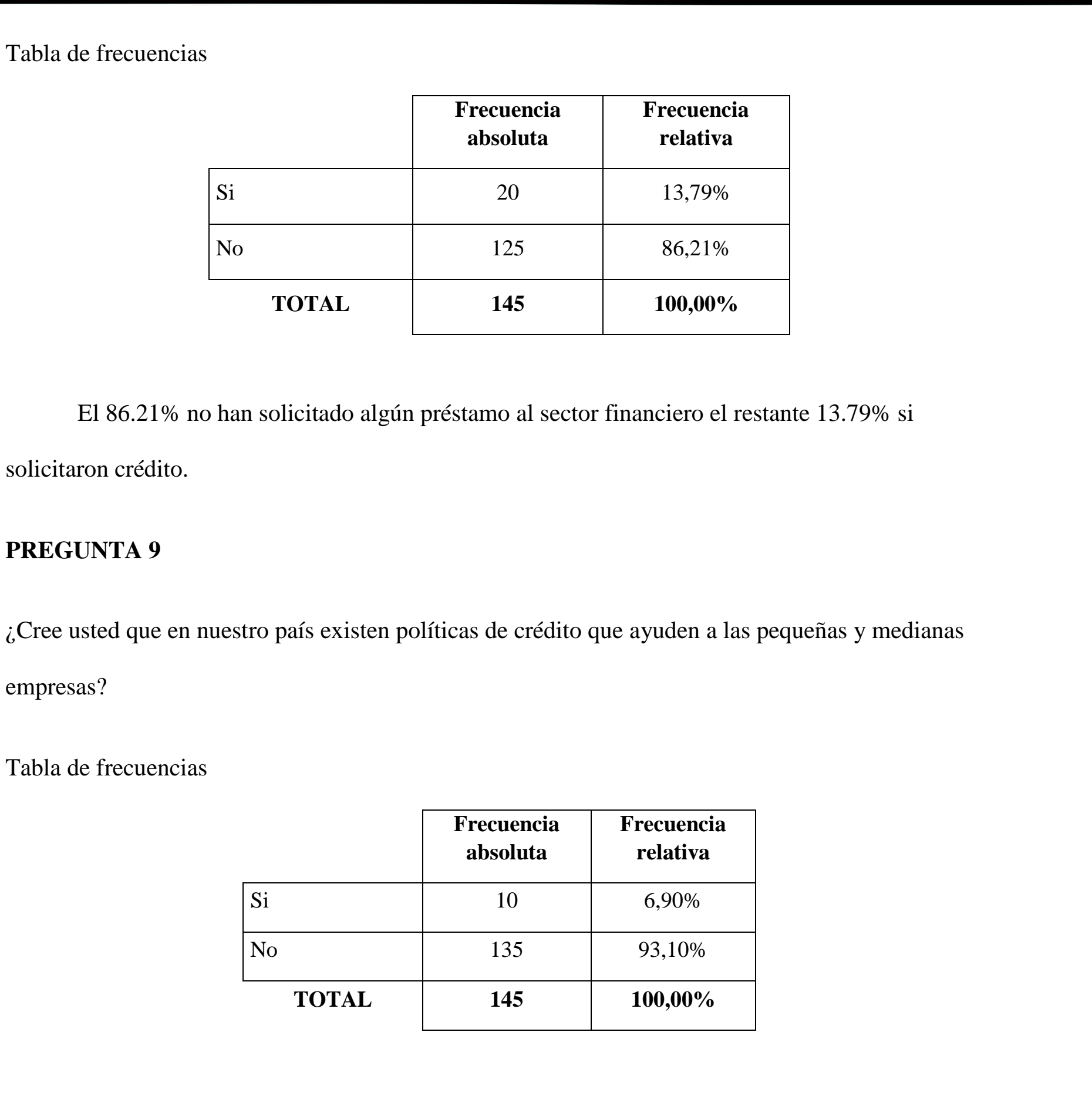

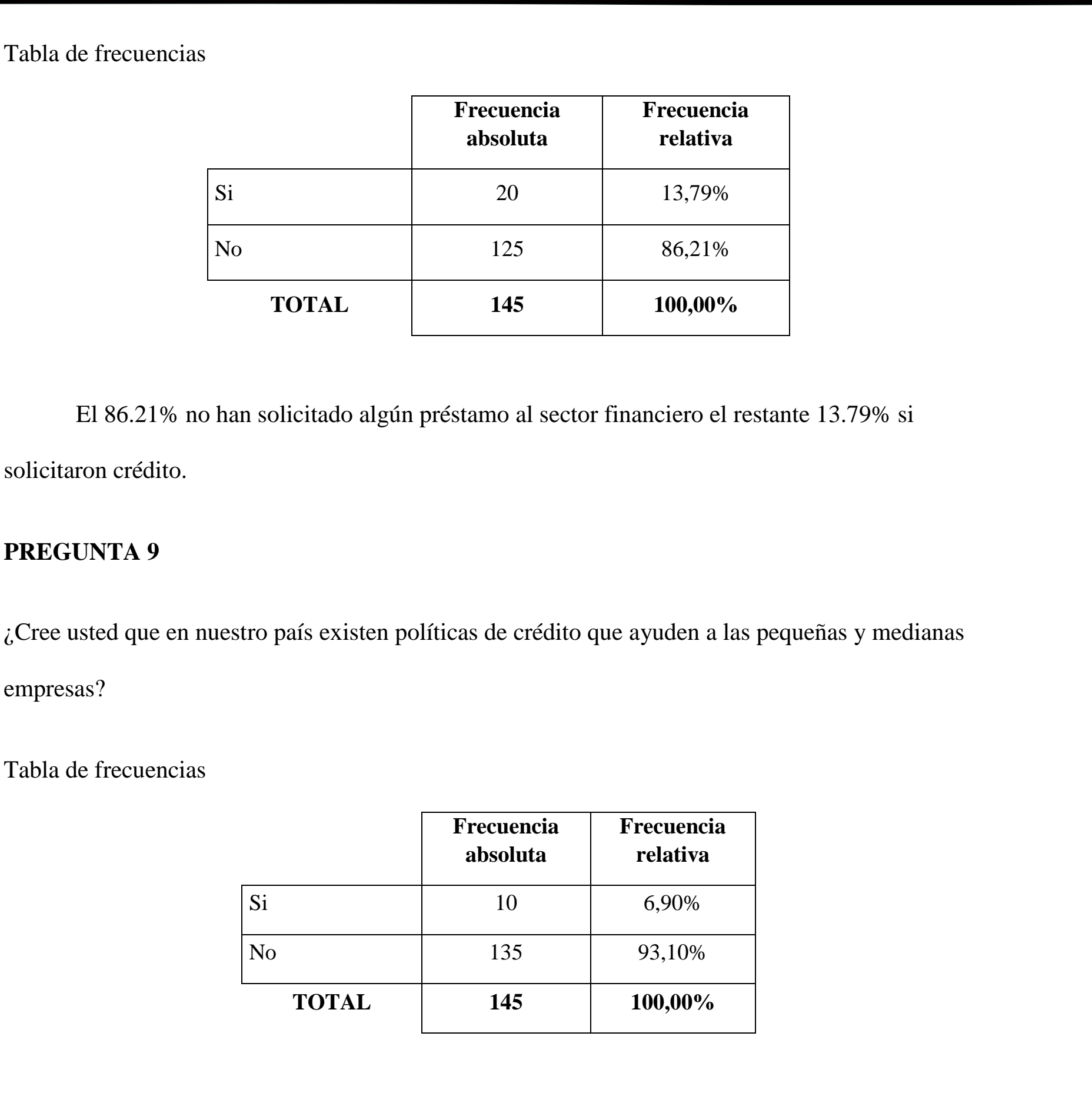

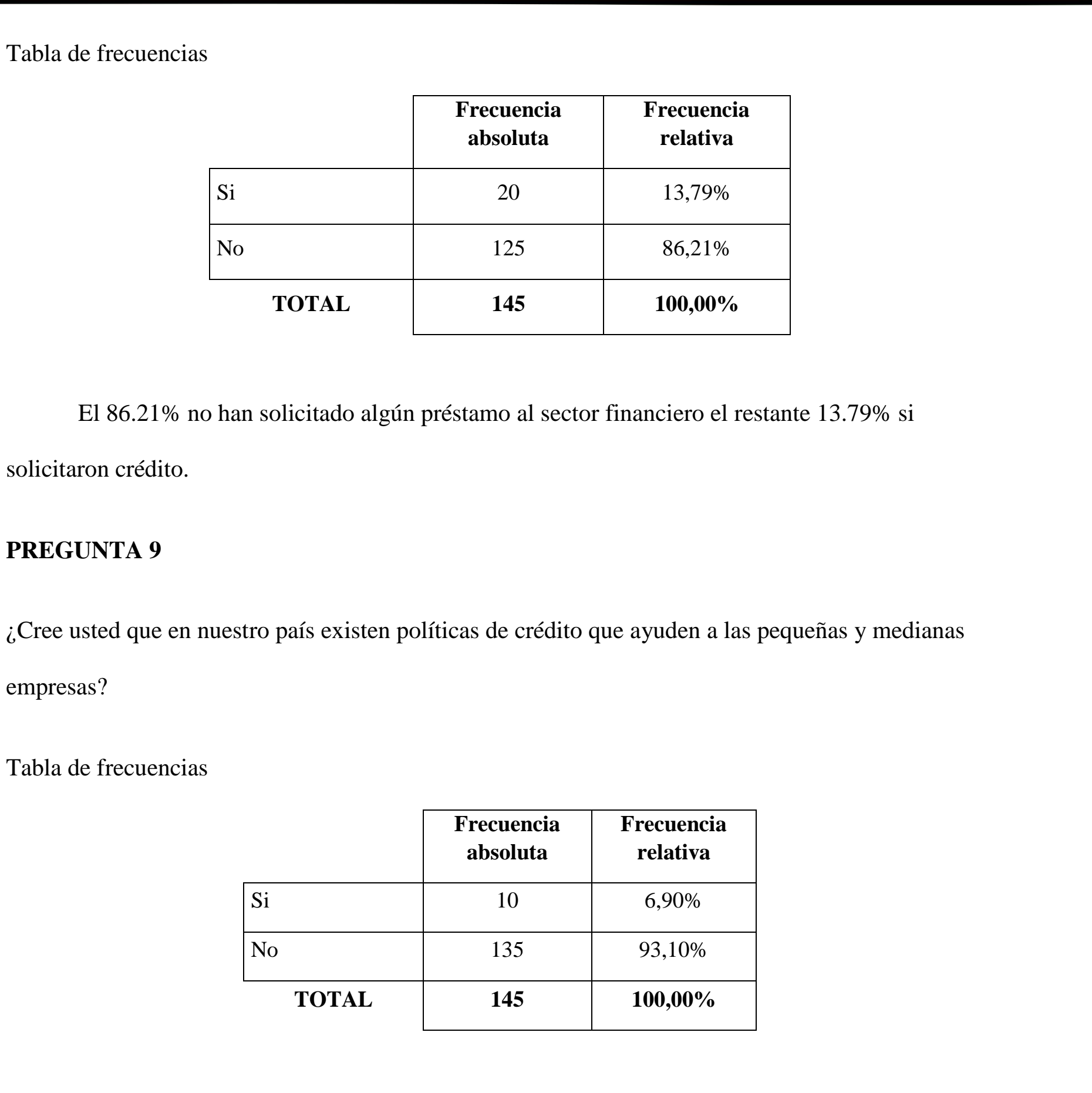

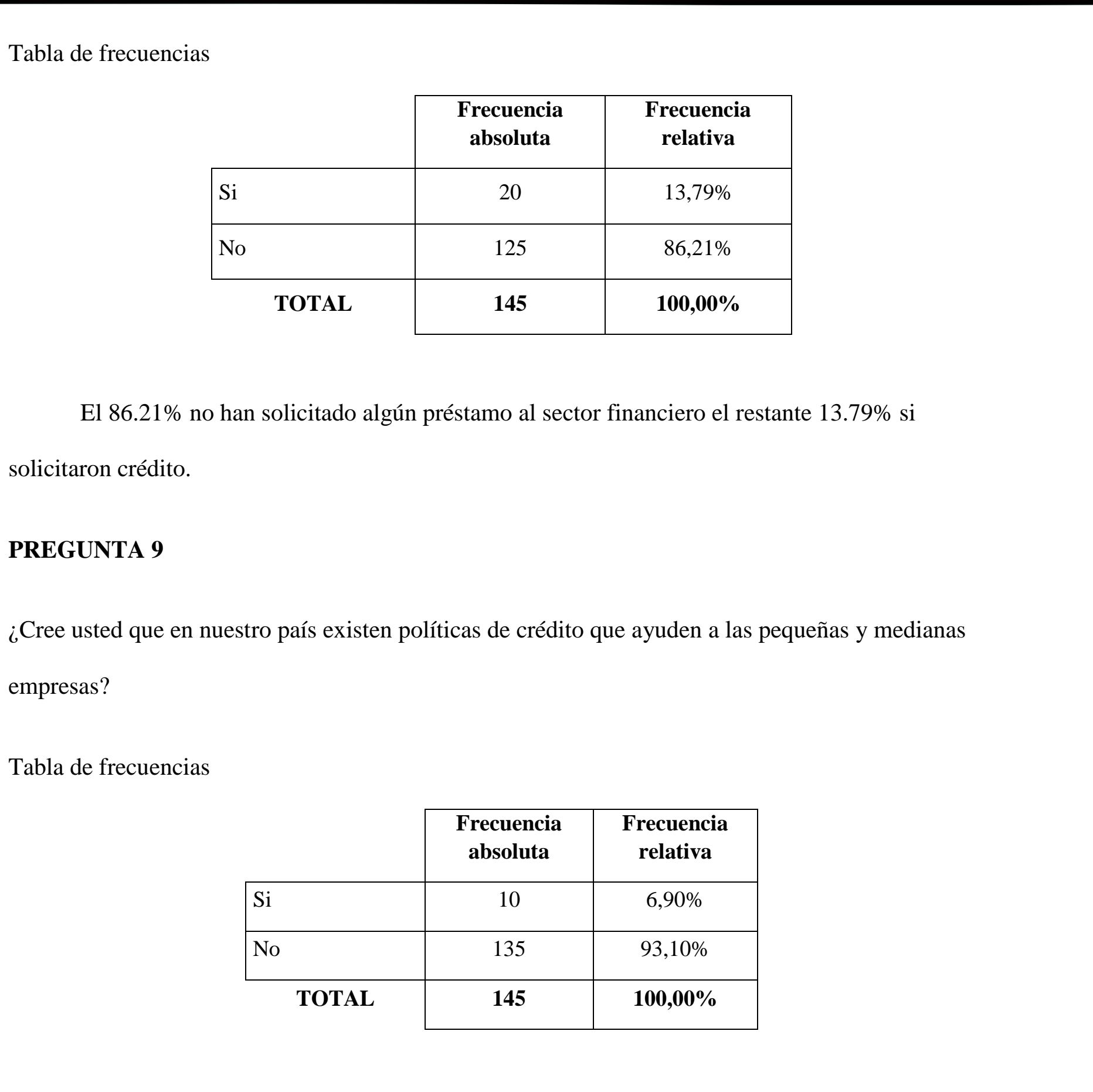

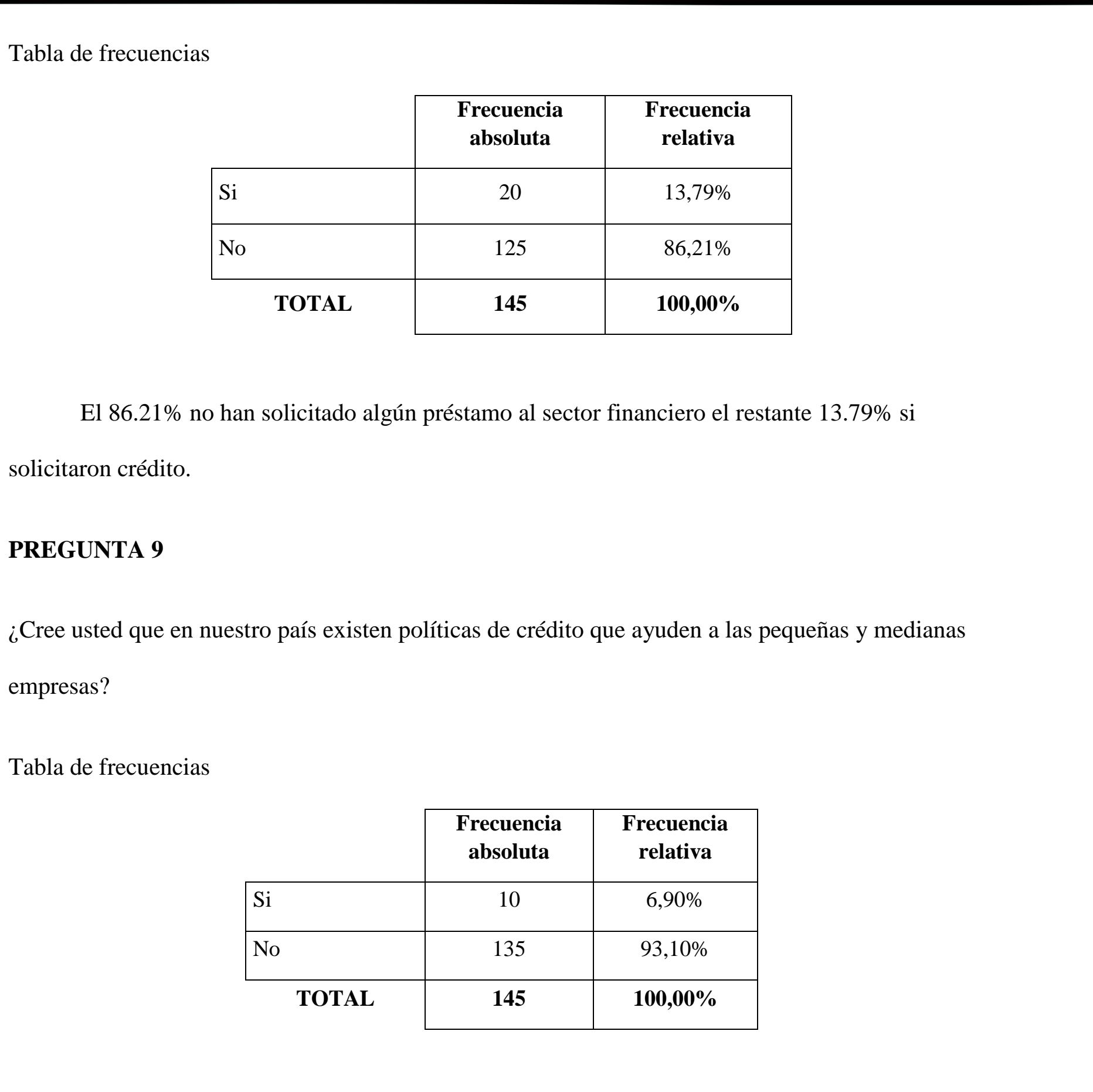

\begin{tabular}{|c|c|c|}
\hline & $\begin{array}{c}\text { Frecuencia } \\
\text { absoluta }\end{array}$ & $\begin{array}{c}\text { Frecuencia } \\
\text { relativa }\end{array}$ \\
\hline $\mathrm{Si}$ & 10 & $6,90 \%$ \\
\hline No & 135 & $93,10 \%$ \\
\hline TOTAL & 145 & $100,00 \%$ \\
\hline
\end{tabular}

El $93.10 \%$ cree que no existe políticas de crédito que les permita desarrollar su negocio, y un 6.90\% si cree que hay política de crédito en nuestro país.

\section{PREGUNTA 10}

¿Conoce usted qué organismos respaldan a los microempresarios? 
Tabla de frecuencias

\begin{tabular}{|c|c|c|}
\hline & $\begin{array}{c}\text { Frecuencia } \\
\text { absoluta }\end{array}$ & $\begin{array}{c}\text { Frecuencia } \\
\text { relativa }\end{array}$ \\
\hline La cámara de la pequeña industria & 3 & $2,07 \%$ \\
\hline La Cámara de Comercio & 2 & $1,38 \%$ \\
\hline Corporación Financiera Nacional & 4 & $2,76 \%$ \\
\hline Ninguna & 136 & $93,79 \%$ \\
\hline TOTAL & 145 & $100,00 \%$ \\
\hline
\end{tabular}

El 93,79\% considera que ninguna institución los respalda, un 2.76\% la CFN seguida por la cámara de la pequeña industria con un $2.07 \%$ y un $1.38 \%$ la cámara de comercio.

\section{PREGUNTA 11}

¿Conoce de los beneficios que le brindan las instituciones financieras como pequeña y mediana empresa?

Tabla de frecuencias

\begin{tabular}{|c|c|c|}
\hline & $\begin{array}{l}\text { Frecuencia } \\
\text { absoluta }\end{array}$ & $\begin{array}{c}\text { Frecuencia } \\
\text { relativa }\end{array}$ \\
\hline $\mathrm{Si}$ & 31 & $21,38 \%$ \\
\hline No & 114 & $78,62 \%$ \\
\hline TOTAL & 145 & $100,00 \%$ \\
\hline
\end{tabular}

El 78.62\% de los entrevistados no conoce de los beneficios que brindan las instituciones financieras para desarrollar el negocio, y un $21.38 \%$ si conocen. 


\section{PREGUNTA 12}

¿Qué le recomendaría a las instituciones financieras para facilitar los trámites de los préstamos a la pequeña y mediana empresa?

Tabla de frecuencias

\begin{tabular}{|c|c|c|}
\hline & $\begin{array}{c}\text { Frecuencia } \\
\text { absoluta }\end{array}$ & $\begin{array}{c}\text { Frecuencia } \\
\text { relativa }\end{array}$ \\
\hline Mayor agilidad & 27 & $18,62 \%$ \\
\hline Reducir los intereses & 31 & $21,38 \%$ \\
\hline Más plazo para pagar & 41 & $28,28 \%$ \\
\hline Masinformación & 17 & $11,72 \%$ \\
\hline Menos requisitos & 29 & $20,00 \%$ \\
\hline TOTAL & 145 & $100,00 \%$ \\
\hline
\end{tabular}

Un 49.66\% de los entrevistados dicen querer más plazo para pagar y menos interés, un $38.62 \%$ quieren menos requisitos y mayor agilidad en los tramites, el $11.72 \%$ que le den más información.

\section{PREGUNTA 13}

¿Qué institución financiera en Babahoyo cree usted que financia mejor a los emprendedores? 
Tabla de frecuencias

\begin{tabular}{|c|c|c|}
\hline & $\begin{array}{c}\text { Frecuencia } \\
\text { absoluta }\end{array}$ & $\begin{array}{c}\text { Frecuencia } \\
\text { relativa }\end{array}$ \\
\hline Banco Nacional de Fomento & 52 & $35,86 \%$ \\
\hline Banco de Guayaquil & 32 & $22,07 \%$ \\
\hline Banco Internacional & 28 & $19,31 \%$ \\
\hline Banco Bolivariano & 12 & $8,28 \%$ \\
\hline Corporación Financiera Nacional & 7 & $4,83 \%$ \\
\hline Cooperativa El Sagrario & 14 & $9,66 \%$ \\
\hline TOTAL & 145 & $100,00 \%$ \\
\hline
\end{tabular}

El 35.86\% de los entrevistados tiene la percepción que el Banco de Fomento es la institución que mejor financia a los empresarios, seguido por el Banco de Guayaquil con un $22.07 \%$.

\section{Conclusiones.}

El 74.48\% de las Pymes en Babahoyo tienen hasta cuatro (4) empleados, seguida por el 4.14\% que tienen de cinco (5) hasta diez (10) empleados y un 3.45\% tienen de quince (15) a más empleados.

Al consultar a los entrevistados si conocían que las instituciones financieras otorgaban créditos a las Pymes, el 61.38\% dijo si conocer.

También se les consulto si conocían que los organismos de crédito solicitan planes de negocio para el otorgamiento de los préstamos, el $86.90 \%$ de los entrevistados dijeron no conocer.

Se les consultó a los entrevistados si desearía recibir un crédito por parte de alguna institución financiera el $75.86 \%$ dijeron que si desearían. 
Implementación de un modelo para elaborar planes de negocios para las PYMES acorde a las exigencias de los organismos de crédito

Al preguntarles que si los organismos de crédito les concediesen un crédito, en que lo invertirían un $70.34 \%$ dijo en desarrollar el propio negocio y el $29.66 \%$ en pagar deudas

En cuanto a la agilidad de los organismos en otorgar un crédito, el $75.86 \%$ manifestaron que es complicado y un $24.14 \%$ consideran que son rápidos

Se les consulto si les gustaría recibir información de cómo solicitar crédito el $88.97 \%$ manifestó que si le gustaría recibir información, un 7.59\% son indiferentes

El 86.2\% no han solicitado crédito en ninguna institución

El 93.10\% cree que no existe en el país una política de crédito que les permita mantenerse en el mercado

Un 93.79\% de los entrevistados consideran que ninguna institución financiera los respalda, un $2.76 \%$ si considera que la Corporación Financiera Nacional los respalda

Se les solicitó que indicaran lo que les recomendaría a los organismos financieros para el otorgamiento de crédito, un $49.66 \%$ dijeron querer más tiempo de plazo para pagar la deuda y que reduzcan los interés, el $38.62 \%$ solicitan menos requisitos y mayor agilidad en los tramites finalmente el $11.72 \%$ quieren más información sobre los requisitos y trámites que hay que hacer.

\section{Propuesta de aplicación}

Nuestra propuesta está enfocada hacia el desarrollo e implementación de un modelo para elaborar planes de negocios para las Pymes acorde a las exigencias de los organismos de crédito en el Cantón Babahoyo, Provincia de Los Ríos. Se trata de un negocio en marcha, con una experiencia acumulada con más de diez (10) años en el mercado de los servicios de: capacitación en el área de la informática, comercialización de computadoras, suministro, partes y piezas 


\section{El desarrollo de la propuesta tiene tres enfoques que son:}

El primer enfoque tiene relación a la conceptualización de las diferentes etapas de un plan de negocio.

El segundo enfoque es la aplicación de la teoría indicada en el apartado anterior en una Pyme que, como se indicó anteriormente, actualmente está compitiendo en el mercado de la informática, es decir, es un caso real, el crédito que solicitará al Banco Bolivariano es de \$12,220.00, el mismo que se utilizará en adecuar el actual local, contratar dos personas y artículos para la venta.

El tercer enfoque es la planificación para la implantación de un cronograma de capacitación, dirigido a todas y cada de las Pymes afiliadas a la Cámara de Comercio así como también a las afiliadas a la Cámara de la Pequeña Industria. Los cursos se impartirán a los diferentes sectores económicos.

\section{Bibliografía.}

AVELlANEDA, Carmenza, "Diccionario de Términos Financieros", México, Editorial Limusa, Segunda Edición, 326 páginas, año 2002.

BURBANO, Ruiz Jorge, "Presupuestos”, Colombia, 1era. Edición, Mc Graw Hill, año 2000.

COSS BU, Raúl: “Evaluación de proyectos de inversión”. Departamento de Ingeniería Industrial ITESM. 2 a. Edición, Editorial LIMUSA, año 2000.

FREUND, Johan E. - Gary A. Simon. “Estadística Elemental”, octava edición, 2005.

GERBIER, Jean. “Organización de Administración de Empresas”, 2004.

HILLIER, Lieberman, “Introducción a la Investigación de Operaciones”, Tercera Edición, 2005.

HOLMES, A. “AuditoríasPrincipios y Procedimientos”. México, Editorial Limusa. 1994.

ILDEFONSO Grande Esteban, "Marketing de los Servicios", Madrid, 3era. Edición, Editorial ESIC, año 2003.

KOENING Carlos Lcdo. “Técnicas de Marketing”, Guayaquil, 1a. Edición. Año 2001.

KOTLER, Phillip, “Dirección de Mercadotecnia”, Editorial México, IV Edición, año 2002. 
Implementación de un modelo para elaborar planes de negocios para las PYMES acorde a las exigencias de los organismos de crédito

MARINILLO, Guerrero Jorge J. Dr., "Instituto Nacional de Oncología y Radiobiología". Calle 29 esquina a E, El Vedado, Ciudad de La Habana, Cuba, año 2000.

MOLINA, Axel Jansson: "Formulación y Evaluación de Proyectos de Inversión", Santiago de Chile, Ediciones Universidad Tecnológica Metropolitana, año 2002.

MURRIER, G. "Planeación y Control de Mercado", EE.UU. Editorial El Conejo, II Tomo, año 1999.

NOEL, Ramírez David., "Empresas competitivas, una estrategia de cambio para el éxito", México, 1era. Edición, Mc Graw Hill Ediciones, año 1998.

OROZCO, Cadena José Dr. "Contabilidad General”, Quito, Tercera Edición, Editor: Productora de Publicación, año 2004.

PHILLIP, Kotler. “Dirección de Mercadotecnia”. - IV Edición, Editorial México 2004.

VILAR P, Tenorio F., "Impacto de la tecnología en el ejercicio de la medicina moderna". Ejercicio actual de la medicina, pp. 99-105.

NAVIA A. ADOLFO. "Manual de Mercadeo”. Ecuador primera edición. Julio del 2009.

NAVIA A. ADOLFO. “Marketing Operacional”. Ecuador primera edición. Agosto del 1995.

NAVIA A. AOLFO. “Como elaborar el Plan Estratégico de Marketing”. Ecuador en proceso de publicación. Julio el 2010. 